\title{
Thirty-Day Mortality After Curative and Palliative Anti-Cancer Treatment: Data Interpretation and Lessons for Clinical Implementation
}

This article was published in the following Dove Press journal: Cancer Management and Research

\author{
Emad Tashkandi $\mathbb{D}^{1,2}$ \\ Bassam Basulaiman (iD ${ }^{3}$ \\ Waleed Alghareeb ${ }^{3}$ \\ Faris Hamadi iD ${ }^{3}$ \\ Anas Alghamdi ${ }^{3}$ \\ Faez Albabakri iD ${ }^{2}$ \\ Redhwan Alshabi ${ }^{2}$ \\ Mohammad Jaffal ${ }^{2}$ \\ Abdulrahman Albaradie 2 \\ Ruqayya Azher ${ }^{4}$ \\ 'Umm AlQura University, College of \\ Medicine, Makkah, Saudi Arabia; ${ }^{2}$ King \\ Abdullah Medical City, Oncology Center, \\ Makkah, Saudi Arabia; ${ }^{3}$ Comprehensive \\ Cancer Center, Medical Oncology, King \\ Fahad Medical City, Riyadh, Saudi Arabia; \\ ${ }^{4}$ Umm Al-Qura University, Community \\ Medicine Department, Makkah, Saudi \\ Arabia
}

Correspondence: Emad Tashkandi Umm AlQura University, College of Medicine, 3829, Aljamiah, Makkah, Saudi Arabia

Tel +96655529006I

Email Tashkandi.e@kamc.med.sa
Purpose: Despite advancements in cancer therapeutics, mortality and morbidity due to anticancer treatments still occur but are not frequently reported. We aimed to report the 30-day mortality and morbidity of all curative and palliative anti-cancer treatments.

Patients and Methods: Adults with solid and hematological malignancies from two large cancer centers in Saudi Arabia, irrespective of the cancer stage and treatment type, were included in this retrospective observational study.

Results: Between December 1, 2019 and February 29, 2020, 1694 patients from King Abdullah Medical City in Makkah and King Fahad Medical City in Riyadh were included in the study. Among them, $77.5 \%$ were younger than 65 years of age; $72.8 \%$ were female; the prevalence of obesity, diabetes, and hypertension was $35 \%, 34 \%$, and $28 \%$, respectively; and $66.5 \%$ of patients had breast and gastrointestinal cancers. Fifty-nine $(3.5 \%)$ patients died within 30 days of receiving anti-cancer treatment. Of them, $9(0.3 \%)$ were treated with curative intent, and $50(3 \%)$ were treated with palliative intent.

Conclusion: Our results emphasize the need to address preventable metabolic changes and implement innovative, predictive, preventive, and personalized medicine (PPPM) approaches focusing on patient profiles. Reporting the 30-day outcomes of all anti-cancer treatments will also allow the identification of factors underlying mortality and morbidity and lead to an improvement in oncological outcomes via innovative programs designed to improve clinical decision-making.

Keywords: cancer, chemotherapy, morbidity, individual outcome, predictive preventive personalized medicine

\section{Introduction}

In Saudi Arabia, the incidence of cancers has increased by ten-fold in the past 30 years. ${ }^{1}$ The most common cancer in Saudi Arabia is breast cancer, followed by colorectal cancer, and the incidence of cancer is higher among females than among males. $^{2}$ The proportion of deaths due to cancer has also increased in the past 20 years, becoming the third leading cause of death in Saudi Arabia. ${ }^{3}$ Chemotherapy agents, which have been shown to improve the survival of cancer patients and their symptoms, have been increasing in number over the past few decades. ${ }^{4-6}$ Caring for patients with cancer is challenging; to improve clinical decision-making, oncologists need to weigh the risks and benefits of anti-cancer treatments and identify factors that could predict treatment-related morbidity or mortality. Despite their occurrence, chemotherapy-related deaths (CRDs) are not frequently monitored or 
reported, and thus, there is no international estimate of the rate of anti-cancer treatment-related mortality.

In a previous retrospective study, Ohe et al retrospectively studied the risk factors for CRDs among lung cancer patients and found that $2.3 \%$ of patients died from chemotherapy-related toxicity. ${ }^{7}$ In the context of small cell lung cancer (SCLC), ${ }^{8}$ reported a chemotherapy-related mortality rate of $5 \%$, secondary to sepsis. Finally, a retrospective study including 267 patients aged $\geq 60$ years, with intermediate or high-grade Non-Hodgkin's lymphoma, and receiving cyclophosphamide-doxorubicinvincristine-prednisone protocol reported a CRD rate of $13 \%{ }^{9}$

Currently, there is no international estimate of standard anti-cancer treatment-related mortality rates. However, based on previous studies, preliminarily establishing an average mortality rate of $5 \%$ (with variations between 3-9\% mortality rate), as a reference, allows for comparisons to be made between different institutions. ${ }^{10}$ The proportion of patients dying within 30 days of receiving anti-cancer treatments may be linked to poor clinical decisions. Thus, 30-day post-treatment mortality rate might be a useful clinical indicator that fits most anti-cancer treatment protocols. Further, the 30-day period allows for recovery from most acute therapy-related adverse events.

No regional or international reports have previously evaluated the 30-day mortality and morbidity following the administration of curative or palliative anti-cancer treatment. Thus, the aim of this study was to report the 30day mortality and morbidity of all curative and palliative anti-cancer treatments.

\section{Patients and Methods}

\section{Study Design and Population}

The Institutional Research Ethics Board at King Abdullah Medical City approved this retrospective observational study. Each patient was consented to collecting research data once the hospital file is created. Therefore, the IRB waved the patients consents in a retrospective study that does not expose patients to high risks. Patient data confidentiality was maintained, and the declaration of Helsinki was followed.

The target population included adults aged $\geq 18$ years who were histologically diagnosed with cancer, irrespective of the cancer stage and class of anti-cancer treatment received. Two out of five comprehensive cancer centers, King Abdullah Medical City (KAMC) in Makkah and
King Fahad Medical City (KFMC) in Riyadh, operated by the Ministry of Health in Saudi Arabia, participated in this study and hence the convenience sampling method was used. Pharmacy administration provided the list of patients who received at least one cycle of anti-cancer treatment in the outpatient setting between December 1, 2019 and February 29, 2020. All potential patients receiving anti-cancer treatment during the study period were included to minimize the potential of confounding bias. A total of 1717 patients were identified, and 1694 patients were eligible and included in the study (1138 patients from KAMC and 556 KFMC). Inclusion criteria were as follows: solid or hematological tumors, received anti-cancer treatments during the study period, and anti-cancer treatments administered orally or parenterally in an outpatient setting. Patients were excluded if they were on regular follow-up or surveillance; received other treatment modalities such as curative surgeries, radiation treatments alone, and best supportive care; or were under treatment with bone-modifying agents such as bisphosphonate and denosumab. The number of patients with missing variables or lost follow-up was $<$ $1 \%$; they were included in the analysis when appropriate.

\section{Study Procedures}

Senior oncology assistants performed a retrospective review of electronic health records (EHRs) to identify subjects who met the eligibility criteria and collected the required data. Collected demographic characteristics included the serial code number, age, gender, and body mass index (BMI). Clinical characteristics included the presence or absence of comorbidities, Eastern Cooperative Oncology Group (ECOG) performance status, type of cancer, and cancer stage. The collected treatment characteristics included protocol name, class (chemotherapy, immunotherapy, hormonal, and targeted therapy), route (intravenous [IV], subcutaneous [SC], and oral), treatment intent (curative or palliative), type of curative treatment (neoadjuvant or adjuvant), line of anti-cancer treatment (first/second/third/fourth-line and beyond), and number of cycles (full data collection provided in Appendix).

Outcome characteristics were 30-day mortality, defined as death within 30 days from day 1 after the last anticancer treatment cycle (excluding road traffic accident and trauma), cause of death, and 30-day morbidity, defined as morbidity within 30 days from day 1 after the last anticancer treatment cycle. Morbidity included 
hospitalizations, emergency room (ER) visits, intensive care unit (ICU) admissions, delay in receiving chemotherapy, and dose reduction. To assess 30-day treatment outcomes patients were followed until March 29, 2020. Cases were analyzed by senior assistants and consultants in oncology to identify the cause of death. The national 30day mortality rate was calculated by dividing the number of patients that received anti-cancer treatment within 30 days of their death by the total number of patients that received anti-cancer treatment in the reported period. If a patient received multiple cycles in this period, 30-day mortality was counted using the most recent cycle of anticancer treatment. Patients receiving multiple treatments in this period were counted only once in the dataset.

\section{Statistical Analysis}

Descriptive analysis of demographic characteristics and outcomes are presented as totals and percentages. Continuous variables are presented as means or medians. Cross-tabulation analysis was performed for categorical variables. All data were checked and analyzed using the Statistical Package for the Social Sciences version 25.

\section{Results}

In the period described, 1694 patients who met the inclusion criteria were included in the analysis. Of these, 1313 $(77.5 \%)$ patients were aged $\leq 65$ years, $1233(72.8 \%)$ were female, and $591(34.9 \%)$ were obese. A total of 1030 $(60.8 \%)$ patients did not have any comorbidities, 664 $(39.2 \%)$ had at least one comorbidity, 228 (34.3\%) had diabetes, 183 (27.6\%) had hypertension, 36 (5.4\%) had ischemic heart disease, and 195 (29.4\%) had other comorbidities. Missing values were $<1 \%$ for all variables (BMI, Stage, ECOG, Class, Route, Intention, 30-day mortality, and 30-day morbidity). Table 1 summarizes participants' demographic characteristics.

In terms of clinical characteristics, 838 (49.5\%) patients had breast cancer, $288(17 \%)$ had gastrointestinal (GI) cancers, 177 (10.4\%) had hematologic malignancies, $123(7.3 \%)$ had lung cancer, $153(9.0 \%)$ had various other types of cancers, and $6.8 \%$ of patients were equally divided between urologic (3.4\%) and gynecologic malignancies $(3.4 \%)$. A total of $784(46.2 \%)$ patients were at stage IV, $475(28 \%)$ at stage III, and 419 (24.7\%) at stages I and II. A total of $1124(66.4 \%)$ patients had an ECOG of 0 or 1 . Table 2 summarizes patients' clinical characteristics.
Table I Demographic Characteristics

\begin{tabular}{|c|c|}
\hline $\begin{array}{l}\text { Characteristics of the } \\
\text { Patients }\end{array}$ & All Patients ( $N=1694), n(\%)$ \\
\hline \multicolumn{2}{|l|}{ Age } \\
\hline$>65$ & $381(22.5)$ \\
\hline$\leq 65$ & $1313(77.5)$ \\
\hline \multicolumn{2}{|l|}{ Gender } \\
\hline Male & $461(27.2)$ \\
\hline Female & $1233(72.8)$ \\
\hline \multicolumn{2}{|l|}{ BMI } \\
\hline Underweight & III (6.6) \\
\hline Normal & $494(29.3)$ \\
\hline Overweight & $492(29.1)$ \\
\hline Obese & $591(35.0)$ \\
\hline \multicolumn{2}{|l|}{ Co-morbidities } \\
\hline Yes & $664(39.2)$ \\
\hline No & $1030(60.8)$ \\
\hline \multicolumn{2}{|l|}{ Main co-morbidities } \\
\hline DM & $228(34.3)$ \\
\hline HTN & $183(27.6)$ \\
\hline IHD & $36(5.4)$ \\
\hline DVT & $22(3.3)$ \\
\hline Others & $195(29.4)$ \\
\hline
\end{tabular}

Note: Data are presented as $\mathrm{n}(\%)$.

Abbreviations: BMI, body mass index; DM, diabetes mellitus; HTN, hypertension; IHD, ischemic heart disease; DVT, deep vein thrombosis.

Regarding anti-cancer treatments, 960 (56.7\%) patients received chemotherapy, followed by hormonal treatment in $467(27.6 \%)$ patients. A total of 1011 (59.7\%) patients received treatment through IV, while 631 (37.3\%) patients received it orally. A total of $928(54.8 \%)$ patients received treatment with curative intent, whereas 762 (45\%) patients received treatment with palliative intent. Most patients $(1221,72 \%)$ received first-line treatments. Table 3 summarizes the characteristics of anti-cancer treatments.

A total of $59(3.5 \%)$ patients died within 30 days of receiving anti-cancer treatments. Of these, $9(0.3 \%)$ patients received potentially curative anti-cancer treatment and 50 (3\%) patients received palliative treatment. Only 9 cases had missing data for 30-day mortality. Causes of death included disease progression in $35(71.4 \%)$ patients, sepsis in 10 $(20.4 \%)$ patients, pneumonia in $2(4.1 \%)$ patients, and bleeding and other reasons in $1(2.1 \%)$ patient.

Meanwhile, an analysis of 30-day morbidity revealed that a total of $508(30.1 \%)$ patients developed morbidity 
Table 2 Clinical Characteristics

\begin{tabular}{|l|l|}
\hline $\begin{array}{l}\text { Characteristics of the } \\
\text { Patients }\end{array}$ & All Patients (N= 1694), n (\%) \\
\hline Diagnosis & $838(49.5)$ \\
\hline Breast & $288(17.0)$ \\
Gastrointestinal & $177(10.4)$ \\
Hematological & $57(3.4)$ \\
Urological & $58(3.4)$ \\
Gynecological & $123(7.3)$ \\
Lung Cancer & $153(9)$ \\
Other & \multicolumn{2}{|l|}{} \\
\hline Stage & $419(25.0)$ \\
\hline I-II & $475(28.3)$ \\
III & $784(46.7)$ \\
IV & \multicolumn{2}{|l}{} \\
\hline ECOG & $566(33.5)$ \\
\hline O-I &
\end{tabular}

Note: Data are presented as $\mathrm{n}(\%)$

Abbreviation: ECOG, Eastern Cooperative Oncology Group performance status.

within 30 days of receiving anti-cancer treatment. Of these, $211(12.5 \%)$ patients received potentially curable anti-cancer treatment and $297(17.9 \%)$ received palliative treatment. Only 4 cases had missing data for 30-day morbidity. Morbidity was related to ER visits in 280 (32.4\%) patients, hospitalization in $209(24.2 \%)$ patients, delay of chemotherapy in $175(20.3 \%)$ patients, dose reduction in $174(20.2 \%)$ patients, and ICU admission in $25(2.9 \%)$ patients. Tables 4 and 5 summarize the 30-day mortality and morbidity outcomes.

\section{Discussion}

The Kingdome of Saudi Arabia (KSA) has initiated a major investment and transformation named "Vision 2030," by which the country aims to undergo fundamental structural changes in all sectors and transform the healthcare system. With an expected population of 45 million by 2030, KSA has the largest and fastest growing population among the Arabic Gulf Countries. Unlike Western countries, which have an aged population, almost $70 \%$ of the population in KSA is younger than 65 . Further, females comprise $43 \%$ of the Saudi population. ${ }^{11,12}$

Because of globalization, metabolic morbidities typical of Western countries have spread to the rest of the world. In KSA, the prevalence of diabetes among adults is $35 \%$, making it the second highest in the Middle East and the
Table 3 Anti-Cancer Treatment Characteristics

\begin{tabular}{|c|c|}
\hline $\begin{array}{l}\text { Characteristics of the } \\
\text { Patients }\end{array}$ & All Patients ( $N=1694), \mathrm{n}(\%)$ \\
\hline \multicolumn{2}{|l|}{ Class: } \\
\hline Chemotherapy & $960(56.7)$ \\
\hline Hormone Therapy & $467(27.6)$ \\
\hline Targeted Therapy & $167(9.9)$ \\
\hline Immunotherapy & $87(5.1)$ \\
\hline Other & II (0.6) \\
\hline \multicolumn{2}{|l|}{ Route: } \\
\hline IV & $1011(60.0)$ \\
\hline Oral & $631(37.4)$ \\
\hline SC & $44(2.6)$ \\
\hline \multicolumn{2}{|l|}{ Intention: } \\
\hline Curative & $928(54.8)$ \\
\hline Palliative & $762(45)$ \\
\hline \multicolumn{2}{|c|}{ Line of palliative therapy: } \\
\hline Ist line & $|22|(72)$ \\
\hline 2nd line & $268(16.4)$ \\
\hline 3rd line & $89(5.4)$ \\
\hline 4th line and beyond & $57(3.5)$ \\
\hline Number of cycles (mean) & 5 cycles \\
\hline
\end{tabular}

Notes: Data are presented as $\mathrm{n}(\%)$ for categorical variables. Mean for continuous variable

Abbreviations: IV, intravenous; SC, subcutaneous.

seventh worldwide. ${ }^{13}$ In our study, we found that $34 \%$ of cancer patients had diabetes. The prevalence of hypertension has been reported to be $26 \%{ }^{14}$ and in our study, we found that $28 \%$ of cancer patients had hypertension. Obesity has a reported prevalence of $29 \%,{ }^{15}$ and in our study, we found that $35 \%$ of cancer patients had obesity. Thus, there is a clear trend toward increased metabolic changes among cancer patients. Such trend can be attributed to lifestyle changes, physical inactivity, diet, eating habits, family history and genetics, as well as cancer or complications from anti-cancer treatments.

Further, we also found a changing profile of cancer cases in Saudi Arabia compared with the rest of the world, with a higher proportion of cancer cases among women, and with breast $(50 \%)$ and GI cancers (17\%) being the most common. Such finding, which can be attributed to metabolic changes, and particularly obesity, is in line with the results of an epidemiological study which included seven countries and reported a higher proportion of cancers attributable to increasing levels of BMI. This observation was most pronounced in the Middle East and 
Table 4 Summary of 30-Day Mortality and Morbidity Rates by Curative and Palliative Intent

\begin{tabular}{|l|l|l|l|l|}
\hline \multirow{2}{*}{} & \multicolumn{2}{l|}{$\begin{array}{l}\text { 30-Day Mortality } \\
\text { (n=1685) }\end{array}$} & \multicolumn{2}{l|}{$\begin{array}{l}\text { 30-Day Morbidity } \\
\text { (n=1690) }\end{array}$} \\
\cline { 2 - 5 } & Yes & No & Yes & No \\
\hline $\begin{array}{l}\text { Curative } \\
\text { Palliative }\end{array}$ & $\begin{array}{l}50(3.5 \%) \\
\text { Total }\end{array}$ & $915(54.3 \%)$ & $211(12.5 \%)$ & $717(42.4 \%)$ \\
$79(3.5 \%)$ & $1626(42.2 \%)$ & $297(17.9 \%)$ & $465(27.5 \%)$ \\
$508(30 \%)$ & $1182(69.9 \%)$
\end{tabular}

generally more rapid in females (in Saudi Arabia $9.2 \%$ in females and $4.0 \%$ in males) than those in the United States (9.5\% and 3.5\%), and (United Kingdom $8.2 \%$ and 4.4\%). ${ }^{16}$ Additionally, the International Agency for Research on Cancer linked excess body weight with increased risk of certain cancer types, including breast, gastrointestinal, gynecologic, urologic, hematologic, and thyroid cancers. ${ }^{17}$ Based on our findings, breast and GI cancers seem to be the most frequently diagnosed cancers among women. The increase in the number of diagnosed cases and stable or lower mortality are likely due to improvements in diagnostic and treatment. Of note, we observed that nearly half of all included patients $(47 \%)$ had metastatic disease and two-thirds (66.7\%) had good performance status (ECOG 0-1). This emphasizes the need to promote a healthy lifestyle and implement effective and innovative preventive, screening and predictive strategies. Treatment approaches should be personalized and focus on patient profiles, particularly in cases of breast

Table 5 Summary of Causes of 30-Day Mortality and Morbidity

\begin{tabular}{|l|l|}
\hline \multicolumn{2}{|l|}{ Causes of 30-Day Mortality (N=59, 3.5\%) } \\
\hline Disease progression & $35(71.4)$ \\
Sepsis & $10(20.4)$ \\
Pneumonia & $2(4.1)$ \\
Bleeding & $1(2.1)$ \\
Other & $1(2.1)$ \\
\hline Causes of 30-day morbidity* (N=508, 30\%) \\
\hline ER visits & $280(32.4)$ \\
Hospitalizations & $209(24.2)$ \\
Delay of chemotherapy & $175(20.3)$ \\
Dose reduction & $174(20.2)$ \\
ICU admission & $25(2.9)$ \\
\hline
\end{tabular}

Notes: Data are presented as $\mathrm{n}(\%)$. *Morbidity includes any of the following: hospitalization, emergency room (ER) visits, intensive care unit (ICU) admissions, delay in receiving chemotherapy, and dose reduction. Therefore, some variables may not add up to $100 \%$. and colorectal cancer, as they represent two-thirds of cancer cases in our study $(67 \%)$.

In our study, more than half $(57 \%)$ of the patients received chemotherapy, with IV route being the most common administration route $(60 \%)$. There is currently no international estimate of the standard 30-day mortality and morbidity secondary to anti-cancer treatments and, at a national level, we also found no studies addressing this issue in Saudi Arabia. In this context, a national benchmark would drive the demand for improving cancer outcomes related to anti-cancer treatments. Results from our population-based study revealed a 30-day mortality of $3.5 \%$ for all anti-cancer treatments. This mortality rate, which could be established as the national standard for Saudi Arabia, was found to be lower than that of previous reports from cancer centers in Australia, England, and New Zealand, which included outpatient systemic chemotherapy for adults with solid and hematologic tumors and reported 30-day mortality rates of 5.6\% for Australia, $4 \%$ for England, and 5.2\% for New Zealand. ${ }^{18-21}$ This could be explained by the younger population in our study than in previous studies.

Furthermore, in our study, we noted that the 30-day mortality after receiving potentially curative anti-cancer treatments was $0.5 \%$, while that of patients treated palliatively was 3\%. The Royal Marsden Hospital in the United Kingdom reported a crude 30-day mortality rate of $8.1 \%$. More specifically, they reported mortality rates of $0.5 \%$ for curative chemotherapy for breast cancers, and $1.5 \%$ for GI cancers. $^{22}$ The Systemic Anti-Cancer Therapy dataset, which is collated by Public Health England and includes 23,228 patients with breast cancer and 9634 patients with non-SCLC, reported a 30-day mortality rate of 3\% for lung cancer patients receiving potentially curative chemotherapy and $10 \%$ for those receiving palliative chemotherapy. In contrast to the previously mentioned studies, which only focused on a subset of cancer types (breast, GI, and lung cancer), our study reported the mortality of all anticancer treatments, and more specifically, analyzed treatments with curative or palliative intent. The population of this study is comparable to that of the Royal Marsden Hospital as $67 \%$ of our population included breast and GI cancer patients.

In this study, we reported a 30-day morbidity rate of $30.1 \%$ for all anti-cancer treatments, including $12.5 \%$ of patients treated with curative intent and $17.9 \%$ of patients treated with palliative intent. The significant increase in the 30-day morbidity of anti-cancer treatments suggests 
that oncologists should pay attention to select the best regimen, dose, schedule, and follow-up, all of which should be coupled with an appropriate healthcare system and quality indicators to identify patients who need continuous support, such as day care, home care visits, telemedicine, and further medications to prevent such morbidity. Additionally, it is important to discuss these findings with patients and their relatives to minimize the burden of anti-cancer treatments and, more specifically of palliative anti-cancer treatments.

This study has several strengths. First, unlike previous studies, we described the 30-day mortality and morbidity rates for all anti-cancer treatments with curative and palliative intent. Second, we included all classes of anticancer treatments such as chemotherapy, immunotherapy, targeted therapy, and hormonal therapy in our analysis, along with the most common routes of administration, such as IV, SC, and oral. Finally, we also included a diverse population of cancer patients, including patients with solid tumors and hematological malignancies.

Our study also has some limitations, such as its retrospective nature and the relatively small number of patients included. Further, since all the patients were from Saudi Arabia, the generalizability of the results to other countries may be limited. Another important limitation of this study is the existence of differences in demographic, clinical, and tumor characteristics compared with other studies. In this study, most patients were younger than 65 years of age and women with breast cancer, thus limiting the generalizability of our results to all cancer types. However, we tried to avoid such differences by inviting more centers to participate. Finally, some patients were excluded from the study due to having missing data. This limitation could be overcome through adequate reporting by physicians as well as through the use of EHRs and electronic prescriptions regularly audited by quality teams.

Current and future innovative approaches, such as preventive, predictive, and personalized medicine (PPPM/3P $)^{23}$ need to be implemented to address modifiable and nonmodifiable risk factors for breast and colorectal cancer, the most frequently diagnosed cancer in our population. Stakeholders, educators, healthcare professionals, and the general population need to increase their efforts to address preventable metabolic changes as they have a substantial link with and are major contributors of the cancer burden. Liquid biopsy can be used for the early detection of cancer, which in turn improves the chances of curative outcomes. Meanwhile, circulating tumor cells can be used to identify metastatic disease. Individualized patient profiling can be achieved through the use of innovative and advanced technologies such as molecular and genomic markers and multi-omics. ${ }^{24}$ Such advanced technologies have positive implications for the quality of cancer treatment but also have an associated cost and burden on patients, the healthcare system, and governments. Thus, to select the appropriate tests and treatments, oncologists need to have the relevant knowledge. ${ }^{25}$ The rapid development of various omics technologies will advance the discovery of new cancer therapeutics capable of achieving meaningful outcomes based on prognostic stratification and tailored to individualized patient profiling. ${ }^{26}$

Future studies should include larger patient populations, have a longer follow-up, and involve more geographically diverse cancer centers. Further, an important research aim is to understand the link between PPPM with mortality and morbidity. Moreover, the use of various omics technologies for the identification of new therapeutics and prognostics could also be a potential area of research.

Reporting the 30-day outcomes of all anti-cancer treatments, as done in this study, will allow the identification of factors underlying 30-day mortality and morbidity and lead to an improvement in cancer care via programs designed to improve clinical decision-making. It will also help provide supportive services to ensure high-quality care for cancer patients. Further, oncologists will be able to use the standard 30-day outcomes as a benchmark to facilitate an informed discussion with cancer patients. Our study highlights the importance of incorporating innovative PPPM approaches for the improvement of cancer outcomes. Overall, the results from this study contribute to existing literature by providing an estimate of the mortality and morbidity of all anti-cancer treatments, whether curative or palliative in intent. Such estimates can also be used to compare outcomes between different cancer centers.

\section{Conclusion}

In conclusion, we need to address preventable metabolic changes and implement innovative, predictive, preventive, and personalized medicine (PPPM) approaches focusing on patient profiles.

The results presented in this study are key as, without a national 30-day outcome benchmark, it is not possible to maintain or improve care for patients on anti-cancer treatments. Further, 30-day mortality reports will allow identification of cancer centers with the highest mortality and 
morbidity, which in turn can facilitate the review of their anti-cancer treatments and healthcare systems, and act as an appropriate quality indicator for the improvement of health care outcomes.

\section{Acknowledgments}

We thank all senior assistants and oncologists who participated in this project. Also, we would like to thank Editage (www.editage.com) for English language editing.

\section{Author Contributions}

All authors made substantial contributions to conception and design, acquisition of data, or analysis and interpretation of data; took part in drafting the article or revising it critically for important intellectual content; agreed to submit to the current journal; gave final approval of the version to be published; and agree to be accountable for all aspects of the work.

\section{Disclosure}

The authors report no conflicts of interest in this work.

\section{References}

1. Althubiti MA, Nour Eldein MM. Trends in the incidence and mortality of cancer in Saudi Arabia. Saudi Med J. 2018;39(12):1259-1262. doi:10.15537/smj.2018.12.23348

2. Bazarbashi S, Al Eid H, Minguet J. Cancer incidence in Saudi Arabia: 2012 data from the Saudi cancer registry. Asian Pacific J Cancer Prev. 2017;18(9):2437-2444. doi:10.22034/APJCP.2017.18.9.2437

3. Tyrovolas S, El Bcheraoui C, Alghnam SA, et al. The burden of disease in Saudi Arabia 1990-2017: results from the Global Burden of Disease Study 2017. Lancet Planet Heal. 2020;4(5):e195-e208. doi:10.1016/S2542-5196(20)30075-9

4. Fossati R, Confalonieri C, Torri V, et al. Cytotoxic and hormonal treatment for metastatic breast cancer: a systematic review of published randomized trials involving 31,510 women. J Clin Oncol. 1998;16(10):3439-3460. doi:10.1200/JCO.1998.16.10.3439

5. Morgan GW, Ward R, Barton M. The contribution of cytotoxic chemotherapy to 5-year survival in adult malignancies. Clin Oncol. 2004;16(8):549-560. doi:10.1016/j.clon.2004.06.007

6. Chabner BA, Roberts TG. Chemotherapy and the war on cancer. Nat Rev Cancer. 2005;5(1):65-72. doi:10.1038/nrc1529

7. Ohe Y, Yamamoto S, Suzuki K, et al. Risk factors of treatment-related death in chemotherapy and thoracic radiotherapy for lung cancer. Eur $J$ Cancer. 2001;37(1):54-63. doi:10.1016/s0959-8049(00)00350-6

8. Radford JA, Ryder WDJ, Dodwell D, Anderson H, Thatcher N. Predicting septic complications of chemotherapy: an analysis of 382 patients treated for small cell lung cancer without dose reduction after major sepsis. Eur J Cancer. 1993;29(1):81-86. doi:10.1016/09598049(93)90581-Y

9. Gómez H, Hidalgo M, Casanova L, et al. Risk factors for treatmentrelated death in elderly patients with aggressive non-Hodgkin's lymphoma: results of a multivariate analysis. J Clin Oncol. 1998;16 (6):2065-2069. doi:10.1200/JCO.1998.16.6.2065
10. Banner R. Benchmark 30-day mortality rate needed. Clin Oncol. 2009;21(9):733-734. doi:10.1016/j.clon.2009.07.007

11. Healthcare Transformation Strategy _ Final. Available from: https://webcache.googleusercontent.com/search? q=cache: M1 uuetchv5UJ:https://www.moh.gov.sa/en/Ministry/vro/ Documents/Healthcare-Transformation-Strategy.pdf $+\& \mathrm{~cd}=13 \& \mathrm{hl}=$ en\&ct $=$ clnk\&gl=sa. Accessed October 20, 2020.

12. Kingdom of Saudi Arabia Healthcare Overview; 2018. Available from: https://webcache.googleusercontent.com/search? $\mathrm{q}=$ cache:1tM3HhopaRcJ:https://www.colliers.com/-/media/files/emea/ uae/case-studies/2018-overview/ksa-healthcare-overview-thepulse -8th-edition.pdf $\% 3$ Fla $\% 3$ Den-gb $+\& c d=2 \& h l=e n \& c t=c l n k \& g l=s a$. Accessed October 20, 2020.

13. Abdulaziz Al Dawish M, Alwin Robert A, Braham R, et al. Diabetes mellitus in Saudi Arabia: a review of the recent literature. Curr Diabetes Rev. 2016;12(4):359-368. doi:10.2174/ 1573399811666150724095130

14. Al-Nozha MM, Abdullah M, Arafah MR, et al. Hypertension in Saudi Arabia. Saudi Med J. 2007;28(1):77-84.

15. Alqarni M. A review of prevalence of obesity in Saudi Arabia. J Obes Eat Disord. 2016;2(2). doi:10.21767/2471-8203.100025

16. Arnold M, Leitzmann M, Freisling H, et al. Obesity and cancer: an update of the global impact. Cancer Epidemiol. 2016;41:8-15. doi:10.1016/j.canep.2016.01.003

17. Lauby-Secretan B, Scoccianti C, Loomis D, Grosse Y, Bianchini F, Straif K. Body fatness and cancer - viewpoint of the IARC working group. $N$ Engl J Med. 2016;375(8):794-798. doi:10.1056/ NEJMsr1606602

18. Gilbar PJ, McPherson I, Aisthorpe GG, Kondalsamy-Chennakes S. Systemic anticancer therapy in the last 30 days of life: retrospective audit from an Australian Regional Cancer Centre. J Oncol Pharm Pract. 2019;25(3):599-606. doi:10.1177/1078155217752077

19. Khoja L, McGurk A, O’Hara C, Chow S, Hasan J. Mortality within 30 days following systemic anti-cancer therapy, a review of all cases over a 4 year period in a tertiary cancer centre. Eur $J$ Cancer. 2015;51(2):233-240. doi:10.1016/j.ejca.2014.11.011

20. Ang E, Newton LV. Thirty-day mortality after systemic anticancer treatment as a real-world, quality-of-care indicator: the Northland experience. Intern Med J. 2018;48(4):403-408. doi:10.1111/ imj. 13618

21. Dahiana Pulgar B, Nicolás Yáñez B, Francisco Ortega G. Mortality within 30 days of receiving systemic chemotherapy at a regional oncology unit. Rev Med Chil. 2019;147(7):887-890. doi:10.4067/ S0034-98872019000700887

22. O'Brien MER, Borthwick A, Rigg A, et al. Mortality within 30 days of chemotherapy: a clinical governance benchmarking issue for oncology patients. $\mathrm{Br} J$ Cancer. 2006;95(12):1632-1636. doi: $10.1038 /$ sj.bjc. 6603498

23. Kucera R, Pecen L, Topolcan O, et al. Prostate cancer management: long-term beliefs, epidemic developments in the early twenty-first century and 3PM dimensional solutions. EPMA J. 2020;11:399-418. doi:10.1007/s13167-020-00214-1/Published

24. Golubnitschaja O, Polivka J, Yeghiazaryan K, Berliner L. Liquid biopsy and multiparametric analysis in management of liver malignancies: new concepts of the patient stratification and prognostic approach. Journal. 2018;9:271-285. doi:10.1007/s13167-018-0146-6

25. Janssens JP, Schuster K, Voss A. Preventive, predictive, and personalized medicine for effective and affordable cancer care. EPMA Journal. 2018;9:113-123. doi:10.1007/s13167-018-0130-1

26. Lu M, Zhan X. The crucial role of multiomic approach in cancer research and clinically relevant outcomes. EPMA J. 2018;9 (1):77-102. doi:10.1007/s13167-018-0128-8 


\section{Publish your work in this journal}

Cancer Management and Research is an international, peer-reviewed open access journal focusing on cancer research and the optimal use of preventative and integrated treatment interventions to achieve improved outcomes, enhanced survival and quality of life for the cancer patient.
The manuscript management system is completely online and includes a very quick and fair peer-review system, which is all easy to use. Visit http://www.dovepress.com/testimonials.php to read real quotes from published authors.

Submit your manuscript here: https://www.dovepress.com/cancer-management-and-research-journal 